\title{
Activating ERK Family Gene Mutation
}

National Cancer Institute

\section{Source}

National Cancer Institute. Activating ERK Family Gene Mutation. NCI Thesaurus. Code C160877.

A mutation in a ERK family gene that encodes a constitutively active MAP family serine/threonine-protein kinase and results in aberrant activation of its downstream pathways. 